\title{
Magnetic resonance, magnetisation transfer, and diffusion weighted imaging correlates of optic nerve, brain, and cervical cord damage in Leber's hereditary optic neuropathy
}

The association between Leber's hereditary optic neuropathy and multiple sclerosis has encouraged the search for susceptibility genes in multiple sclerosis. This symbiotic relation is reversed in the paper by Inglese et $a l^{1}$ (this issue, pp 444-449) in which magnetic resonance (MR) techniques originally developed for studying multiple sclerosis were applied to patients with Leber's hereditary optic neuropathy. The authors aimed to quantify the amount of optic nerve damage, and, more interestingly, detect the degree of pathological change in the brain, in a disease often thought in its isolated form to be highly tissue specific for the optic pathways.

Leber's hereditary optic neuropathy is probably the most studied disease caused by mitochondrial DNA (mtDNA) mutations. It usually results in bilateral and severe visual loss in young adults, with a preponderance in men. It has been associated with other neurological syndromes, the best described as resembling multiple sclerosis and occurring mainly in female patients, often with a family history of Leber's hereditary optic neuropathy (also called Harding's disease). ${ }^{2}$ Advances in mitochondrial genetics have facilitated our knowledge of the disease. Most patients with Leber's hereditary optic neuropathy are linked to the "primary" mtDNA mutations at nucleotide positions 11 778, 3460, and 14 484, all located in genes encoding polypeptides found in complex I of the oxidative phosphorylation pathway. However, incomplete penetrance may occur and unaffected family members can be virtually homoplasmic (>95\% mtDNA) for a particular mutation but remain asymptomatic in life. This may be explained by other aetiological influences including environmental factors (for example, tobacco and alcohol use) and the presence of "secondary" mtDNA mutations. Clinically, the primary mutations give rise to slightly different phenotypes, with 11778 (and rarely 3460) being associated with Harding's disease, whereas 14484 is the most benign mutation with the best prognosis for visual recovery (40\%). Recent in vitro functional experiments have perhaps complicated our understanding of the pathogenesis of Leber's hereditary optic neuropathy, with the mutations generating different changes in biochemical markers. ${ }^{3}$ In vivo MR studies have so far produced conflicting results regarding abnormalities within and outside the optic nerve.

Inglese et al employed MR techniques developed in their earlier series on multiple sclerosis, including thin slice conventional $\mathrm{MR}$, non-conventional imaging with magnetisation transfer (MT), and echo planar diffusion weighted imaging (DWI), to investigate a relatively heterogeneous cohort (10 patients with isolated Leber's hereditary optic neuropathy and four with Leber's hereditary optic neuropathy-multiple sclerosis). The first part of this study established the presence of optic nerve atrophy, as confirmed in the past by postmortem histology. The second part demonstrated that widespread microscopic abnormalities occurred in the normal appearing white matter of the brain of patients with Leber's hereditary optic neuropathy. Similar findings have been noted previously in patients with multiple sclerosis. The cause for this microscopic damage remains speculative, as both MT and DWI are rather non-specific markers of structural change. The results of Inglese et al agree with the findings from other MR spectroscopic series that pathology in Leber's hereditary optic neuropathy is not restricted to the optic pathway, ${ }^{4}$ and therefore contradict the conclusions from in vitro biochemical experiments which have detected normal metabolism in brain tissue.

To clarify the issue, future MR series will require techniques more specific to pathology, such as MR spectroscopy, to characterise changes caused by Leber's hereditary optic neuropathy and discriminate it from other diseases known to affect the normal appearing white matter (for example, multiple sclerosis and systemic lupus erythematosus). ${ }^{5}$ Certainly our increasing knowledge of Leber's hereditary optic neuropathy has created the need for further studies to elucidate the underlying mechanisms of this complex, multifactorial disease.

Division of Clinical Neurology, University Hospital, Queen's Medical Centre, Nottingham NG7 2UH, UK

nnxcl@nnnl.med.nottingham.ac.uk

1 Inglese $\mathrm{M}$, Rovaris $\mathrm{M}$, Bianchi $\mathrm{S}$, et al. Magnetic resonance imaging, magnetisation transfer imaging, and diffusion weighted imaging correlates of optic nerve, brain, and cervical cord damage in Leber's hereditary optic neuropathy. I Neurol Neurosurg Psychiatry 2001;70:444-9.

2 Harding AE, Sweeney MG, Miller DH, et al. Occurrence of a multiple sclerosis-like illness in women who have a Leber's hereditary optic neuropathy mitochondrial DNA mutation. Brain 1992;115:979-89.

3 Cock HR, Cooper JM, Schapira AHV. Functional consequences of the 3460-bp mitochondrial DNA mutation associated with Leber's hereditary optic neuropathy. 7 Neurol Sci 1999;165:10-17.

4 Cortelli P, Montagna P, Avoni P, et al. Leber's hereditary optic neuropathy: genetic, biochemical, and phosphorus magnetic resonance spectroscopy study in an Italian family. Neurology 1991;41:1211-15.

5 Rovaris M, Viti B, Ciboddo G, et al. Brain involvement in systemic immune mediated diseases: magnetic resonance and magnetisation transfer imaging study. F Neurol Neurosurg Psychiatry 2000;68:170-7. 